3.

\title{
Der Krebs des Uterushalses in seinen Anfaugsstadien.
}

\author{
Von Prof. Hegar in Freiburg i. Br.
}

Jeder Arzt, welcher sich mit dem Krebs des Gebărmutterhalses zu befassen bat, und Gelegenheit dazu ist leider häufig genug vorhanden, wird sjch die alte Frage wieder aufwerfen, wie wohl das Ding, welches er vor sich bat, im Anfang ausgesehen kabe. Bei den pathologischen Anatomen kann man sich keisen Rath holen. Sie bekommen nur ein noch vorgerückteres Stadium zu Gesicht, als der Klniker. Die Analogie mit anderen Körpertheilen kann nicht als maassgebend betracbtet werden. Die Schildernngen in den Lehrbüchern der Gynakologie lassen sich unschwer als Constructionen erkennen, welche der Analogie und dem jedesmaligen schwankenden Standpunkt der theoretischen Anschaungen entnommen sind. Sie nehmen sich mit der gehörigen differentiellen Diagnose verbrämt ganz stattlich aus. Nur macht es einen eigenthümlichen Eindruck, wenn die Verfasser oft selbst sagen oder zo verstehen geben, dass sie das Anfangsstadium des Uebels nicht geseben baben. Festgestellt ist, dass der Krebs des Uterushalses aus einer papillaren Wucherung seinen Anfang nehmen kano und bezügliche Beobachtungen sind hierüber vorbanden. Allein die bei Weitem grösste Zabl der Erkrankungen hat diesen Ursprung offenbar nicht. Die Mittheilung eines Falles im ersten Stadium möchte deshalb von Interesse sein, insbesondere als ein sehr ausgeprägtes blinisches Bild sich damit verband, welches man sonst nur bei ganz anderer Gewebsalteration findet. Ich.schliesse diesem noch einige andere Beobachtungen an, welche zwar das Bild in vorgerückterem Stadium zeigen, allein doch noch nicht das Bild darstellen, welches man gewöhnlich zu Gesicht bekommt.

Rrebsige Schwellung des Uterushalses mit Vorragen aus der Schamspalte, partieller Inversion der Scheide, vollständig äbnlich dem Allongement du col de l'utérus (Huguier).

Frau G., Multipara, 68 Jahre alt, verlor mit 43 Jahren ihre Menses obne irgend welche Beschwerden. Sie war vollständig gesund bis vor einem Jahre, wo sie ein Gefühl von Druck im Unterleib, Abwärtsdrängen, Brennen in den Genitalien und zeitweise mässigen Blutabgang bemerkte. Seit 14 Tagen bemerkt Pat. einen Vorfall, welcher des Nachts nicht voliständig wieder zurücktritt. Dabei starke, ziehende Schmerzen im Kreuz und Hypogastrium, sobald die Kranke längere Zeit auf den Beinen ist.

Aus der erweiterten Schamspalte ragt etwa $3 \mathrm{Cm}$. weit ein cylindrischer Tumor vor mit einem Durchmesser von $\breve{5} \mathrm{Cm}$. An der Spitze befindet sich der querspaltige Muttermund, dessen nächste Umgebung eine flache Erosion zeigt, kaum so bedeutend, als man sie bei Katarrhen des Cervix vorfindet. Die Scheide ist theilweise invertirt. Vorn dringt der Finger auf 3 , hinten auf $5 \mathrm{Cm}$. weit in den Blindsack derselben ein. Die Blase und der Mastdarm sind bei dem Vorfall unbetheiligt. Die Sonde geht anf $12 \mathrm{~cm}$. in den Uterus ein. In etwa $7 \mathrm{Cm}$. Entfernung rom 
äusseren Muttermund findet sich eine etwas schwieriger za passirende Stelle, wohl das Orif, internum. Diesem entspricht auch die bimanuelle Untersuchung per vaginam, anum und Bauchdecken, welche den Hals enom intumescirt, den Kobrper von andabernd pormalen Dimensionen ergibt. Das Resultat der Untersuchung war ein solches, wie man es häufig bei gutartigen Hypertropbien des Uterushalses antrifft. Verdacht erregte es jedoch, dass das Uebel in so spater Lebenszeit auftrat, besonders da kein Symptom einer früber vorhandenen Affection der Sexualorgane sich gezejgt hatte. Anfallend war ferner der Umstand, dass der. Tumor sich verbaltnissmäsig weich anfühte. Die Grenze zwisehen supravaginalem Theil des Collum und Yaginalportion liess sich, wie leider in vielen ăbnlichen Fallen, nut approximatiw bestimmen. Ich schälale die Lange der Vagmalportion auf etwa $2 \mathrm{Cm}$. In dieser Entfernung rom orif. ext. warde daher der Schnit begonnen und dann trichterformig weiter nach dem Lumen des Cervicalkanats za excidirt. Hierbei fol die Weichbeit des Gewebes noch inebr auf, Die erste Vereingung der Wunde durch Umsäumung der Halsscbleimhat mit dem asseren Wundrand gelang nicht. Die Wunde zeigte lebhafte, fungöse Wucherungen und worde deshalb, nach aunmehr festgestelter milroskopische Diagnose mit dem Ferrum candens cauterisirt. Enge Wochen spatter verliess die Kranke das Hospilat Die Wunde zeigte eine zormal granulirende Fläche von sehr verkleinertem Unfang und mässiger Eiterung,

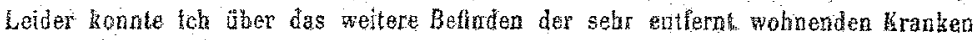
pichts waberes erfuluren.

Das Präparat hat vollständig die Form und Grösse einer gutartig hypertrophischen Vaginalportion. Die Geschwärsbilaung, wenn man einen einfachen Epithelverlust so nennen kann, ist so gross, als man sie bei einfachen Katarrhen des Cervix băufg findę Bề den kthischen Demonstrationen, bel welchen ich gutartige Hypertrophien zum Vergleich zeige, muss ich Acht baben, dass das Carcinom wieder in sein riebtiges Glas zoruekkommt. Die Consiatenz ist auch an dern Spirituspräparat noels weich. Die mikrostopisebe Untersuchung ergab eine ansses-

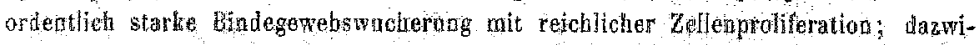
schen elnzelne eingestreute Epithelbaufen, einfach in Lüchen des gewacherten Biode. gewebes siturt, ohoe selbständige alveoläre Begrenzung Diese Nester finden sich

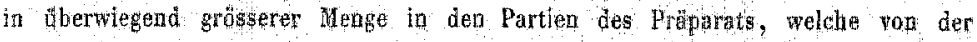
freien Flache entferth lagen, sicher in derselben Menge fo dem Gewebe, welches der Mucosa nicht angehort, als in dieser. En Auspang van den Drüsen hasst sich nicht nachweisen und die Entwichelung der Papullen an der freien erodinten Flache to nicht bedouteader, als man sie bei anderen Erogionen anch findet.

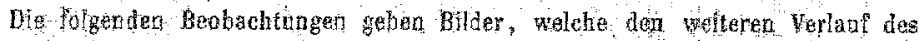
Iebels darstellen, obgleich nicht gesagt sein soll, dass niebt auch andere Entwirchelungen und Vebergang formen existiren

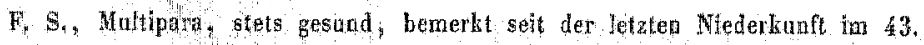
Lebengabre, dass di Menes stâker, lange dauernd and mit Schmerzed im liretrz und Leib verbunden sind. Sle ist jetzt $5 a$ Jahre alt. Seit einem balbea datre

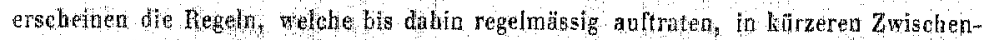
räumen. Auch tn den Interialen finden sich Blotabgange Sonstger Ausluss tst

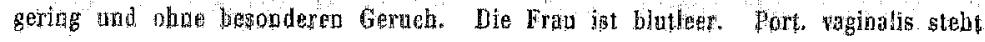


nach vorn, hintere Lippe gleichmässig geschwellt, verlärgert, breit und dick, nirgends böckerig, mit vollständig intacter Scbleimbaut, ausgenommen eine Stelle. In der Mitte am Rande des querspaltigen Muttermundes erhebt sich etwa 2-3 Mm. über das Niveau eine lebhaft rothe, himbeerartige, etwa $1 \frac{1}{2} \mathrm{Cm}$. im Durchmesser baltende Wucherung mit granulirter Oberfläche, welche einen graulichen, eitrigen Schleim absondert. Die vordere lippe ist kurz, verdünnt, scharfrandig und legt sich wie ein Saum auf die Anschwellung der hinteren Lippe auf. Der Vteraskörper ist in mässiger Retroversion durch das hintere Scheidengewölbe zu betasten. $\mathbf{E r}$ ist vergrössert, weich, schmerzhaft gegen Druck. Der Vorsehlag eines operativen Eingriffs wurde nicht aceeptirt. Die Kranke starb etwa $\frac{3}{4}$ Jahre später. Sonst konnte ich über den weiteren Verlauf Nichts erfahren.

Diese Beobachtung bietet grosse Aehnlichkeit mit derjenigen, welche uns in dem trefflichen Werke: Traité pratique des Maladies de l'Utérus etc. par Boivin et Dugès p. 27. pl. 28. Fig. 1 u. 2 überliefert ist. Nur sitzt die Affection bier in der vorderen Lippe, hat eine mehr knollige Form und die fungöse Ulceration liegt entfernter vom Muttermund. An der carcinomatösen Natur des Uebels wird Niemand zweifeln, weleher die zweite Figur, den Zerfall der Anschwellung darstellend, betrachtet.

Frau R., 70jährige, aber sehr rüstige Bauersfrau, hat seit etwa einem balben Jahre Blutungen und fleischwasserähnlichen Ausfluss aus der Vagina. Die hintere Lippe bildet einen etwa wallnussgrossen Knollen, dessen nach dem querspaltigen Muftermund hin gerichtete Oberfläche ein buchtiges Geschwïr mit fungösen Wncherungen von mässigem Umfang zeigt. Die vordere Lippe legt sich als dünner Saum auf den Tumor auf. Auch die höheren Partien der vorderen Cervicalwand zeigen keine Intumescenz. Der Uteruskörper ist klein und beweglich. Der hintere Knoten wird weggeschnitten, worauf man im supravaginalen Theil der hinteren Halswand einen zweiten, vom ersten ziemlich isolirten, kleineren Knollen füblt. Ich entfernte auch diesen, soweit dies möglich war, mit Messer und Scheere und applicirte zur Destruction von Resten das Ferrum candens. Sehr wenig Reaction folgte auf den eingreifenden Act und die Kranke verliess 14 Tage später mit granulirender Wunde die Klinik.

Frau K., 53 Jahre alt, hat 3 Kinder geboren, das letzte vor 22 Jahren. Menses nach der letzten Niederkunft stets regelmässig, auch in den letzten Jahren, nicht copiös, 3 Tage davernd. Seit einem halben Jahre wurde die Periode profuser und hielt länger an. Seit einem Vierteljahre beständig theils rein blutiger, theils bluttingirter, eitrig-jauchiger Ausfluss von üblem Gerucb. Schmerzen werden nicht geklagt. Nur hat die Kranke das Gefühl, als ob sich etwas aus der Scheide bervordränge. Anämisches Aussehen. Klage über Kraftlosigkeit. Scheide weit und faltig. Port. vag. steht tief und nacb vorn. Muttermund nach der Schoossfuge gerichtet, breit, klaffend. Beide Lippen enorm geschwellt, bilden einen ringförmigen Wulst von 5-6 Cm. Durchmesser. Dieser Wulst wird dem Muttermund znnächst durch die ectropiirte, geschwellte, stark geröthete Cervicalmucosa gebildet. Weiter nacb aussen gleicht die Oberfläche dem äusseren Aussehen nach der Scheidenschleimbaut. Schwierig ist die Entscheidung, welcher Theil der Vaginalportion und welcher der supravaginalen Partie des Collum angehört. Ich schătzte die 
Länge des in die Vagina hereinagenden Tumors auf $2 \frac{1}{2}$ Cm. Vom Ansatz des Scheidengewölbes an den Gebärmutterhals aus fühlte man weiter nach hinten einen stark verdickten Cervix, gelangte dann an einen knickungswinkel, hinter welchem der mässig geschwellte Uteruskörper lag. Sämmtliche Theile zeigen einen nicht unbedeutenden Grad von Beweglichkeit. Nach Freilegung der Geschwulst durch Speculum und Hebel zoigte sich die linke Hälfte nnd zwar sowohl der innere Deberzug des Wulste6, die geschwollene Cervicalmucosa, als auch die nach aussen gelegene Schleimbaut der Vaginalportion olne alte Substanzverluste, Die rechte Hatfte dagegen zeigte ein thefes, rissiges Geschwär pach allen Michtungen mit vorquellenden fungösen. Wucherungen. Dock arstreckten sicb die Substanzweriuste fast ausscbliesslich auf die ectropische Mucosa des Cervieallianals und griffen nur wenig in die äassere Schleimbant der Vaginalportion wber, welche auch her thr biassrothes anseben bewahrte. Das Ganze glich einer lartoffel oder falen Frucht, deren Hülle an einer Stelle geplatzt isf, worauf der whgelockerto Inhalt heryorquilit.

Die Exstirpation wurde versucht. Durch einen tief nach den Gerpicalkand gelegten trichterförmigen Schnit, welcher nach aussen etwa $2 \frac{1}{2} \mathrm{Cm}$. oberhall der tiefsten Partie der Geschwolst begann, wurde ein grosser. Theil der Anschwellung excidirt. Allein an allen Stellen des zurückgebleberen Gewebes qualled die zerfallenen Komedonen der Nebbldong, wie kleine eitrige Wäste herwor. Wiederbolte Abtragungen, welche den stapravaginalen Theil des Collam aushollter, fohrten za keinem besseren Restitate. Die Jebhafte Blatung stilte toh durch Costechung. Werzehn Tage spater zeigte sich auf der vorderen Parte dez Wuade eine inaselmussgrosse Excrescenz, auf welche das Ferrum canders aufgesetzt worde Die Kranke batte trata destarken Eingrifs kam gefiebert. Die milkrodtopisehe Untersuchung zeigte the cancroide Structur mit weit vorgeschittenem Zertall.

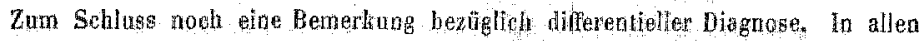
Lehbüchern fndet man die Angabe, dass eine Hrate, bockerige, linolige oder knotige Beschafenheit der Vagialportion das Anfangstadium noteres Vebels charakterisire Noob in dem nenesten Aufsate urgr Caroinema ateri von Gas a. row (Sammlung thinither Yorthge von Volkman No, 18) ist angefubrt, dass

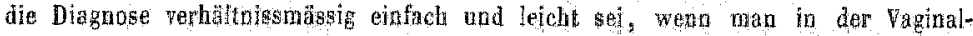

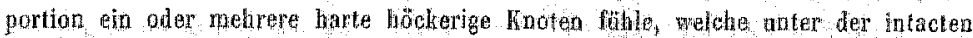

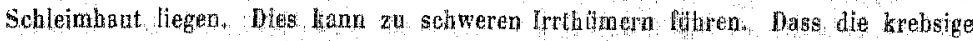

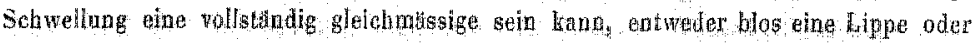
beide exgreifend, ist hinlânglich bekant. Aber weniger herworgehoben ist, dass ganz gatartige Mfumestenzen eine exguisit höclerge und knollige Fom darbieten

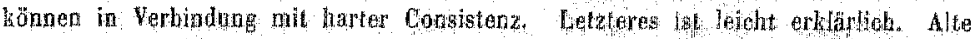

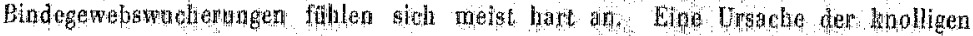

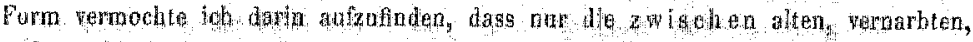

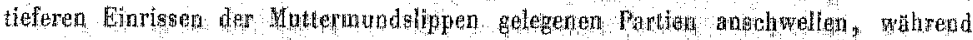

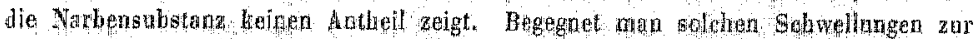

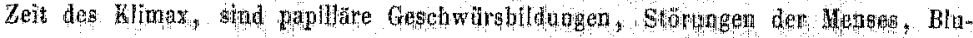

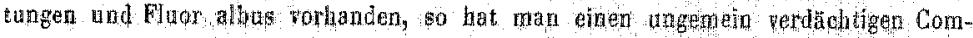

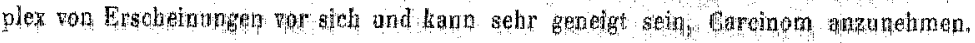

